\title{
TRANSPLANTE NO BRASIL: UM INVESTIMENTO DO SUS
}

\author{
Transplantation in Brazil: a SUS’s investment
}

Genilde Oliveira dos Santos

\section{RESUMO}

As políticas de saúde no Brasil vêm sendo desenvolvidas desde a década de 20, com a criação do Seguro Social, mas somente na década de 30 foram criados os Institutos de Aposentadoria e Pensões (IAP) organizados por categorias profissionais. Na década de 60, o governo unificou os IAP e criou um sistema único, o INPS (Instituto Nacional de Previdência Social). O SUDS (Sistema Unificado e Descentralizado de Saúde), aprovado em 1987, foi o antecessor do SUS (Sistema Único de Saúde). Uma das políticas de saúde atualmente em vigor é gerenciada pelo SNT (Sistema Nacional de Transplantes) e atualmente, mais de $80 \%$ dos transplantes de órgãos no Brasil são financiados pelo SUS. O presente estudo objetiva descrever o processo de doação e transplante de órgãos e tecidos como uma das políticas de saúde no Brasil. Foi realizado estudo descritivo retrospectivo da política de saúde no Brasil, com ênfase nos procedimentos relativos aos transplantes de órgãos e tecidos; os dados analisados foram fornecidos pelo Registro Brasileiro de Transplantes - RBT e pela Central de Notificação, Captação e Distribuição de Órgãos de Sergipe - CNCDO-SE. A partir de dados do RBT, observa-se o crescimento no número de transplantes. O estado de Sergipe, o menor da federação, também conta com o apoio do SUS e vem crescendo aos poucos em número de transplantes, como pôde ser evidenciado através de dados de 2001 a 2008 fornecidos pela CNCDO-SE, com destaque para os anos de 2001, 2003, 2004 e 2007, apesar da falta de alguns recursos, como laboratório de imunogenética. Concluiu-se então com a pesquisa que o Brasil vem aos poucos melhorando em número de doações, conforme registro do RBT com um quantitativo de 3954 transplantes de órgãos sólidos em 2001, passando a 4836 transplantados no ano de 2007; já no ano de 2008, percebe-se uma significativa redução, com registro de apenas 2505 transplantes efetuados no período. Esse fato só comprova que é preciso intensificar o processo de doação através da implementação de disciplinas pertinentes à doação de órgãos e tecidos nas universidades, orientação da população através da mídia, palestras nas escolas visando uma redução efetiva na lista de espera, já que quando se relacionou os dados sobre transplantes efetuados e lista de espera no mesmo período, observou-se um aumento significativo na lista de espera a cada ano, que era de 43581 pessoas em 2001 , passando a 68908 em 2008

Descritores: Política, Saúde Pública, Brasil, Transplantes.

\section{Instituição:}

Unidade de Terapia Intensiva do Hospital Universitário de Sergipe e Central de Transplantes de Sergipe

\section{Correspondência:}

Rua Benjamin Fontes 151, Ed. Malibu - Ap. 502 - Aracaju / SE - CEP: 49045-110 - Brasil Tel.: (79) 32178941

E-mail:d.genilde@ig.com.br

Recebido em: 23.07 .2008

\section{INTRODUÇÃO}

Somente na década de 20 é que se dá a primeira medida concreta, em nível nacional, para a criação do sistema de saúde pública. ${ }^{1}$ Considerados o marco da medicina previdenciária no Brasil, são criados em 1930, os Institutos de Aposentadoria e Pensões (IAPs). ${ }^{2}$

O SUDS (Sistema Unificado e Descentralizado de Saúde), aprovado em 1987, foi o antecessor do SUS (Sistema Único de Saúde). O SUDS surgiu sob a forma de convênios do INAMPS com as Secretarias Estaduais de Saúde. ${ }^{2}$

A Carta Magna de 1988 garante a saúde como direito de todos e um dever do Estado, tendo os usuários acesso igualitário e universal às ações de promoção, prevenção e recuperação da saúde. As instituições privadas têm sua participação de forma a complementar aos serviços do SUS. ${ }^{2}$

Após a criação do SUS, o governo passou a atender a população de forma integralizada, inclusive financiando procedimentos de alta complexidade, como é o caso dos transplantes de órgãos. No decreto que regulamentou a lei (Decreto Lei ñ .2 .268 ), em junho de 1997, 
o Ministério da Saúde criou o Sistema Nacional de Transplante (SNT) e as Centrais de Notificação, Captação e Distribuição de Órgãos (CNCDO), conhecidas como Centrais Estaduais de Transplante e estabeleceu a forma de distribuição de órgãos e tecidos através das listas de espera unificadas. ${ }^{3} \mathrm{O}$ Sistema Público de Saúde financia mais de 95\% dos transplantes realizados no Brasil e também subsidia parte dos medicamentos imunossupressores para todos os pacientes. ${ }^{4} \mathrm{O}$ governo subsidia também procedimentos de alta complexidade, biopsias, exames e em 2008, colocou em vigor a portaria GM 2042 de 25 de setembro, que define a forma de ressarcimento pelo SUS dos procedimentos relativos à retirada de órgãos para transplantes a hospitais não autorizados ou não credenciados pelo SUS.

\section{MÉTODOS}

No presente trabalho, foi realizada uma pesquisa indireta, que utiliza o método descritivo e retrospectivo da Política de Saúde no Brasil, com ênfase no transplante de órgãos. A pesquisa foi desenvolvida com base em artigos científicos sobre o assunto, disponíveis nas bases de dados Scielo e Bireme e do portal do Ministério da Saúde. A partir daí, foi feito um levantamento histórico que percorreu a fase evolutiva da saúde pública brasileira até a organização do transplante no país. Utilizou-se para discussão dados fornecidos pelo Registro Brasileiro de Transplantes - RBT sobre o transplante de órgãos sólidos e tecidos de 2001 a 2008, além de dados atuais do menor estado da federação, Sergipe, fornecidos pela Central de Transplantes do estado referentes aos transplantes de rim, coração e córnea, bem como a representação da evolução da lista de espera de 2001 a 2008, fornecidos pelo RBT.

\section{RESULTADOS E DISCUSSÃO}

A figura abaixo mostra a evolução do transplante (TX) no Brasil de 2001 a 2008, provando que o investimento na política de

Figura 1. Evolução do Transplante no Brasil

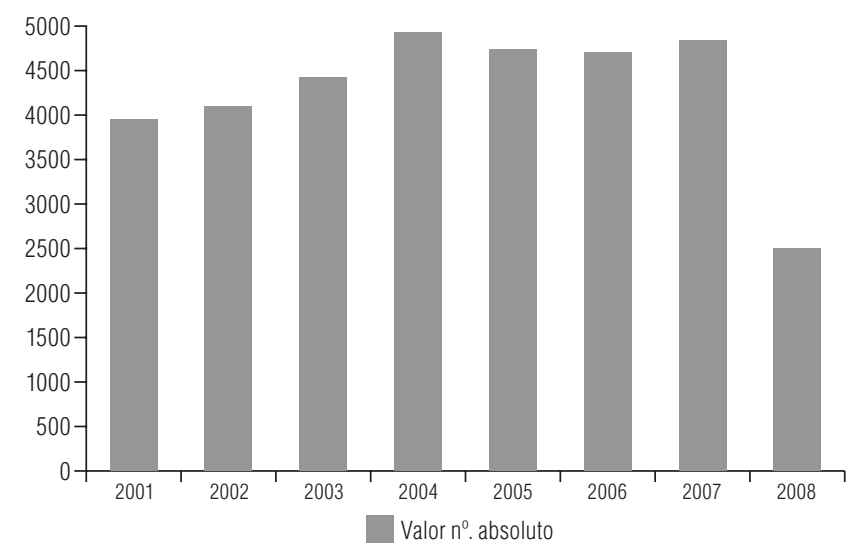

\begin{tabular}{lllllllll}
\hline ANO & $\mathbf{2 0 0 1}$ & $\mathbf{2 0 0 2}$ & $\mathbf{2 0 0 3}$ & $\mathbf{2 0 0 4}$ & $\mathbf{2 0 0 5}$ & $\mathbf{2 0 0 6}$ & $\mathbf{2 0 0 7}$ & $\mathbf{2 0 0 8}$ \\
\hline Valor $\mathrm{n}^{0}$. absoluto & 3954 & 4095 & 4417 & 4927 & 4735 & 4699 & 4836 & 2505 \\
\hline
\end{tabular}

Fonte: RBT

*Dados referentes a órgãos sólidos: coração, fígado, intestino, pâncreas, pulmão e rim

** 2008 Dados referentes ao primeiro semestre transplante vem surtindo efeito e crescendo gradativamente, fato esse que traz benefícios para a população. No entanto, ainda falta muito para que a meta seja atingida, que, de acordo com dados do Registro Brasileiro de Transplantes, RBT "a taxa de doadores efetivos no primeiro semestre de 2008 foi superior em $5 \%$ à do ano passado e $16 \%$ à do primeiro semestre de 2007 ; entretanto, esse aumento foi insuficiente para atingir a taxa prevista para este ano (igual ou superior a 7 pmp)" ${ }^{5}$ Em relação aos transplantes de órgãos, observou-se em comparação com o ano anterior, aumento nos transplantes de coração (18\%), pulmão (9\%), fígado $(5 \%)$ e rim $(3 \%)$, enquanto que o transplante de pâncreas, nas suas três modalidades diminuiu 11\%. Entretanto, as taxas de transplante de coração (1,0 pmp), pâncreas $(0,8 \mathrm{pmp})$ e pulmão $(0,3 \mathrm{pmp})$ são muito baixas em relação à taxa de doadores efetivos (6,5 pmp). No transplante renal houve aumento de 5\% com doador falecido e bqueda de $2 \%$ de doador vivo.

Na figura 2 destaca-se a evolução dos transplantes em Sergipe, o menor estado da federação, desprovido de alguns recursos como laboratório de imunogenética, porém com pequeno aumento no número de transplantes, destacando-se os anos de 2001, 2003, 2004 e 2006.

Figura 2. Transplantes em Sergipe 2001 a 2008

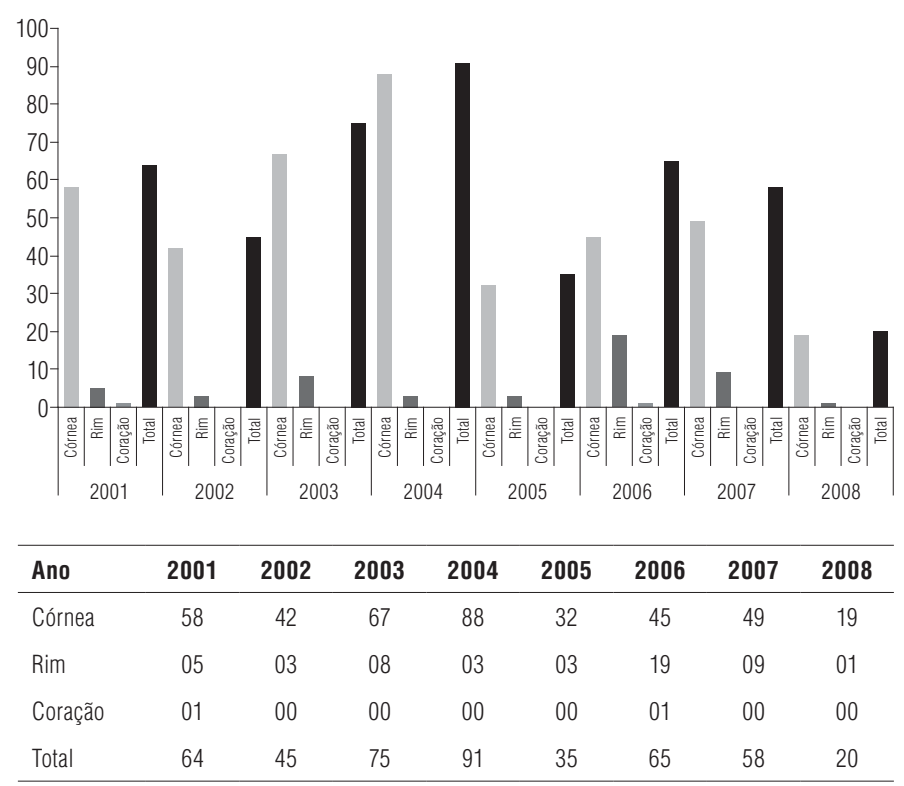

Fonte: CNCDO-SE

Principalmente no Nordeste, devido possivelmente a fatores sócioculturais, há um índice modesto de doações em comparação com o Sul e o Sudeste, isso sem considerar a dimensão geográfica e populacional dessas regiões, onde, segundo dados do Instituto Brasileiro de Geografia e Estatística - IBGE no ano de 2007 a região Nordeste, região que conta com nove estados, possuía uma população estimada em 51.534.406 habitantes. Em contrapartida, a região Sudeste, com quatro estados, tinha população estimada em 77.873.120. ${ }^{6}$ De acordo com dados do RBT, “embora tenha havido crescimento nas taxas de doação e de transplantes de órgãos 
no primeiro semestre de 2008, esse foi inferior ao planejado e as medidas para acelerar esse crescimento devem ser ampliadas". ${ }^{5}$

Na figura 3 foi feita uma relação entre o total de transplantes no Brasil de 2001 a 2008 e a evolução anual de lista de espera no mesmo período para órgãos e tecidos, a exemplo de: coração, córnea, fígado, pâncreas, pulmão rim e rim/ pâncreas, mostrando a discrepância entre transplantes ocorridos e o número de indivíduos que aguardam na lista de espera.

Figura 3
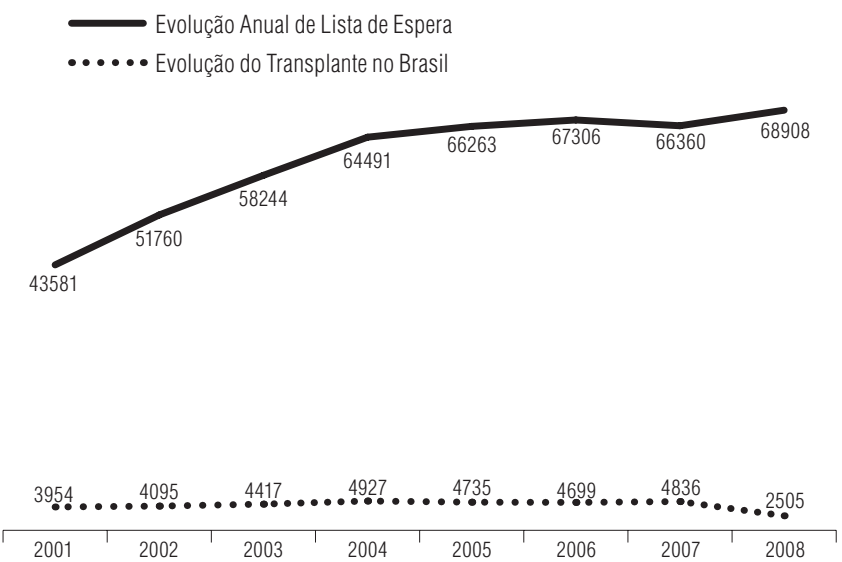

Fonte: RBT

\section{Considerações finais}

A Saúde Pública no Brasil passou por diversas fases na tentativa de promover assistência à população, e, a partir da Constituição de 1988, a saúde passou a ser dever do Estado e um direito do cidadão. Surge o atendimento integralizado à população, inclusive de procedimentos de alta complexidade; o transplante de órgãos e tecidos passa então a fazer parte da política de saúde no Brasil, onde atualmente o SUS cobre mais de $80 \%$ dos procedimentos de transplantes, incluindo o transporte de órgãos e a liberação de imunossupressores.

O Brasil vem aos poucos melhorando em número de doações, e cuidados pós enxerto, conforme registrado pelo RBT, com um quantitativo de 3954 transplantes de órgãos sólidos em 2001, passando a 4836 transplantados no ano de 2007, porém precisa melhorar ainda mais para que se possa ter uma redução efetiva na lista de espera, que, quando equiparadas com o número de transplantes, mostra-se significativamente maior. ${ }^{5}$

Em Sergipe, o menor estado da federação, observa-se sensíveis melhoras em relação à doação de órgãos e tecidos, mas também, como em todo o país, é necessário que especialmente a logística melhore, já que se corre contra o tempo. Além disso, utilizar um laboratório de imunogenética de outro estado é um entrave. Há necessidade de conscientizar melhor a população quanto à importância da doação e o número de vidas que podem ser salvas e, ainda os profissionais da saúde, para que invistam no potencial doador e não os vejam como uma perda de tempo e/ou sobrecarga desnecessária de trabalho.

\section{ABSTRACT}

The Brazilian health policy has been developing since the 20's upon the creation of the social security system, but only in the 30's the Instituto de Aposentadoria e Pensão were created (IAPs) organized by professional categories. In the 60's, Government unified the IAP creating an unique system, (Welfare Department), the INPS (Instituto Nacional de Previdência Social). The SUDS (Sistema Unificado e Descentralizado de Saúde) approved in 1987 was SUS's (Sistema Unico de saúde) predecessor. One of the health policies now in force is managed by SNT (Sistema Nacional de Transplantes) and the organ transplantation in Brazil is nowadays more than $80 \%$ funded by SUS. The present study aims to describe the organ donation process and the organ transplantation as one of the health policies in Brazil. A retrospective descriptive study of the health policies was performed, emphasizing the related organ transplantation procedures; data analyzed was supplied by the Registro Brasileiro de transplantes - RBT and the Central de Captação e Distribuição de Órgãos de Sergipe - CNCDO-SE. Beginning from the RBT data, it was observed the growth in the amount of transplants in the state of Sergipe, the smallest state of the federation, also supported by SUS, which is growing little by little in the amount of transplants, and this can shown through data supplied by CNCDO-SE from 2001 to 2008, particularly in the years of 2001, 2003, 2004 and 2007 in spite of the lack of some resources, as an laboratory of immunogenetic. It was concluded in the research that Brazil is improving its amount of organ donations, according to the RBT registration, with an amount of 3954 transplants of solid organs in 2001, passing to 4836 transplanted in 2007. In 2008, it is noticed a significant reduction of registrations when considering only the 2505 transplants made during the period, and such fact only proves that it is necessary to intensify the organ donation process by applying pertinent discipline comprising the organ donation in universities, orienting the population through the media, lectures in schools aiming to attain an effective decrease on the wait list, since when linking these data to the transplantations performed and the waiting list during that same period, a significant increase is observed on the waiting list each year that belonged to 43581 people in 2001, passing to 68908 in 2008

Keywords: Politics, Public Health, Brazil, Transplants. 


\section{REFERÊNCIAS}

1. Perez LA apud Luz MT. Notas sobre as políticas de saúde no Brasil de "transição democrática" - anos 80. PHYSIS, Revista de Saúde Coletiva, [periódico na Internet]. 1991 [acesso em 15 de maio de 2008];1 (1): 323-31. Disponível em http:॥www.scielo.com.br.

2. Cohn A, Elias PEM. Saúde no Brasil: políticas e organizações de serviços [periódico na internet]. 1996 [acesso em 15 de maio de 2008]; 75:902-4. Disponível em http:bases.bireme.br.

3. Garcia VD. A política de transplante no Brasil. Painel desenvolvido em sessão da Academia Sul - Rio Grandense de Medicina no dia 28 de agosto de 2006. Rev. da AMRIGS [periódico na internet]. Out. a dez. 2006. [acesso em 15 de maio de 2008];50(4):312-20. Disponível em: http//www.amregs.com.br.

4. Rev. Assoc. Med. Bras. Transplante de órgãos no Brasil, [periódico na internet]. Jan. a mar. de 2003 [acesso em 15 de maio de 2008]; 49; (1). Disponível em: http:// www. scielo.br\scielophp.

5 Garcia VD; Registro Brasileiro de Transplantes. Enfim a retomada do crescimento, [periódico na internet] jan a dez 2008 [acesso em 15 de maio de 2008]; ano XIV $\mathrm{n}^{\circ}$ 2. Editorial ano 2008. Disponível em http//www.abto.org.br.

6. Instituto Brasileiro de Geografia e Estatística IBGE. Recenseamento 2007. [homepage na Internet]. Disponível em: http//www.ibge.gov.br/recensseamento. 